\title{
Higher expression of circulating miR-182 as a novel biomarker for breast cancer
}

\author{
PING-YU WANG ${ }^{1,2}$, HAI-TAO GONG ${ }^{3}$, BAO-FENG LI ${ }^{4}$, CHUN-LEI LV ${ }^{4}$, HUAN-TAI WANG ${ }^{3}$, \\ HUI-HUI ZHOU ${ }^{5}$, XIN-XIN LI ${ }^{2}$, SHU-YANG XIE ${ }^{2}$ and BAO-FA JIANG ${ }^{1}$ \\ ${ }^{1}$ School of Public Health, Shandong University, Jinan, Shandong 250012; ${ }^{2}$ Key Laboratory of Tumour Molecular Biology \\ in Binzhou Medical University, Department of Biochemistry and Molecular Biology, Binzhou Medical University, \\ Yantai, Shandong 264003; ${ }^{3}$ Laiyang Central Hospital of Weifang Medical College, Yantai, Shandong 264000; \\ ${ }^{4}$ Shandong China Traditional Medical Affiliated Hospital, Jinan, Shandong 250012; ${ }^{5}$ Department of Pathology, \\ Affiliated Yuhuangding Hospital, Medical College of Qingdao University, Yantai, Shandong 264000, P.R. China
}

Received May 5, 2013; Accepted September 9, 2013

DOI: $10.3892 / \mathrm{ol} .2013 .1593$

\begin{abstract}
MicroRNAs (miRNAs), present in the serum in a stable and reproducible manner, may be used as biomarkers for various diseases. Few studies have previously investigated circulating miRNAs in the peripheral blood of breast cancer (BC) patients. To identify the role of serum miR-182 levels in $\mathrm{BC}$, the present study detected miR-182 levels in the serum of 46 BC patients and 58 controls, by quantitative PCR. The results showed that the serum miR-182 levels in $\mathrm{BC}$ patients were significantly higher compared with the serum of healthy controls $(\mathrm{P}<0.01)$. The miR-182 was also overexpressed in the $\mathrm{BC}$ tissues compared with the para-carcinoma tissues. Furthermore, the serum levels of miR-182 in the estrogen receptor (ER)-positive patients were considerably lower compared with those in the ER-negative patients. The serum levels of miR-182 in the progesterone receptor (PR)-positive patients were also found to be lower compared with those in the PR-negative patients. The current study highlights results consistent with miR-182 as a novel and valuable biomarker for the diagnosis of $\mathrm{BC}$.
\end{abstract}

\section{Introduction}

Breast cancer (BC) is the most common type of cancer in females worldwide. BC originates most commonly from the inner lining of milk ducts or lobules of breast tissue (1), which accounts for $22.9 \%$ of all types of cancer (with the exception of non-melanoma skin cancer) in females and has caused

Correspondence to: Professor Bao-Fa Jiang, School of Public Health, Shandong University, 44 Wenhuaxi Road, Jinan, Shandong 250012, P.R. China

E-mail: wpingyugirl@163.com

E-mail: bjiang@sdu.edu.cn

Key words: miR-182, circulating miRNAs, breast cancer, diagnosis, gene expression
458,503 mortalities worldwide (13.7\% of cancer mortality in females) in 2008. According to the American Cancer Society, almost 230,000 new cases and 40,000 mortalities occurred in the United States in 2011. However, recognized risk factors of BC may be absent in 50-80\% of patients (2), which establishes an increased interest to identify possible risk factors that contribute to BC.

microRNAs (miRNAs) are small ( 22 nucleotides), non-coding RNA molecules. miRNAs, which modulate the expression of targeting genes by post-transcription, are involved in the regulation of various cell processes, including apoptosis, hematopoietic cell differentiation, metabolism, neural development and metastasis $(3,4)$. A number of miRNAs are involved in several types of human cancer, including BC. The majority of previous studies have described the profile of miRNA expression in BC cell lines and primary tumor tissues. For example, increased expression of the miR-191/425 cluster in aggressive $\mathrm{BC}$ cells changes global gene expression profiles, which has a fundamental impact on the progression of BC cells (5). By analyzing the miR-21 expression in $\mathrm{BC}$ tissues, Ozgün et al previously reported that patients with high miR-21 expression levels have a significantly lower disease-free survival than patients with low miR-21 expression levels, which indicates that miR-21 is an indicator of an aggressive $\mathrm{BC}$ phenotype (6). The overexpression of miR-21 increases BC MCF-7 cell growth, migration and invasion, self-renewal and clonogenicity (7). The overexpression of miR-200a protects tumor cells from anoikis and promotes metastases, while inhibition of miR-200a suppresses anoikis resistance in $\mathrm{BC}$ cells (8). The decreased miR-200f expression is likely to increase the expression levels of EMT-transcriptional inducers and may be used as a hypothetical biomarker to assess the occurrence of EMT in BC (9). Let-7, as a tumor suppressor, inhibits the estrogen receptor (ER) $\alpha$-mediated cellular malignant growth in ER-positive BC stem cells (10). The abovementioned studies show that miRNAs are important for the tumorigenesis, migration and invasion of $\mathrm{BC}$.

The apoptotic and necrotic primary tumor discharges miRNAs into the blood circulation, known as circulating 
miRNAs. Therefore, blood contains circulating miRNAs from numerous cells (including tumor cells), which makes it possible to detect miRNAs from specific organs, tissues or cells using surface markers for proper quantification $(11,12)$. Moreover, the circulating miRNAs, resistant to RNase activity, are rare and extremely stable in serum and plasma (13). This stability translates into consistent miRNA expression levels among individuals, which makes serum miRNAs attractive biomarkers for the diagnosis of BCs. However, there have been only a few previous publications investigating circulating miRNAs in the peripheral blood of BC patients (13-16). The present study investigated the levels of miR-182 in the blood serum of BC patients to identify the potential of serum miRNAs as biomarkers for BC.

\section{Materials and methods}

Subjects. The present study was performed at the Inpatient Department of Medical Oncology of Laiyang Central Hospital (Yantai, China). The research protocol was approved by the Medical Ethics Committee of Binzhou Medical University (Yantai, China). All experiments were performed according to the relevant guidelines of the Medical Ethics Committee of Binzhou Medical University.

In total, 46 BC patients, aged 30-79 years, were pathologically diagnosed with $\mathrm{BC}$, for the first time, between May 1st, 2010 and September 30th, 2012. The patients had not received prior chemotherapy. Healthy controls $(n=58)$, came to Laiyang Central Hospital for physical examination between May 1st, 2010 and September 30th, 2012 and were diagnosed without any tumor or physical illness. Prior to inclusion, all the eligible $\mathrm{BC}$ patients and healthy controls provided written informed consent following a full explanation of the study procedures.

Immunohistochemistry. Histological sections (3- $\mu \mathrm{m})$ were deparaffinized in xylene and rehydrated. Antigen retrieval was performed by microwaving the sections in $10 \mathrm{mM}$ citric acid monohydrate. Endogenous peroxidase activity was blocked by $0.5 \% \mathrm{H}_{2} \mathrm{O}_{2}$ treatment. The slides were incubated with appropriate dilutions of the primary antibodies [anti-ER, 1:200; and anti-progesterone receptor (PR), 1:200; ZSGB-BIO, Beijing, China] at $4^{\circ} \mathrm{C}$ overnight. The same procedure was performed for negative controls which were incubated overnight in 1X PBS without antibody. The reaction was visualized by the ABC Kit (ZSGB-BIO) and positive ER and PR status was defined by nuclear staining of $>10 \%$.

miRNA isolation from serum and tissue. Serum samples from the patients and controls were collected between 7:00 and 8:00 a.m. Following centrifugation for $30 \mathrm{~min}$ at 2,650 g, plasma samples were stored at $80^{\circ} \mathrm{C}$. miRNAs were extracted from plasma by the mirVana ${ }^{\mathrm{TM}}$ miRNA isolation kit (Ambion, Carlsbad, CA, USA) according to the manufacturer's instructions. Tissue samples were homogenized in a denaturing lysis solution. Total RNA was extracted from tissue lysis using the TRIzol reagent (Invitrogen Life Technologies, Carlsbad, CA, USA). Then, miRNA was separated from 30-50 mg of total RNA using the Ambion miRNA Isolation Kit (Ambion).
Quantitative PCR ( $q P C R)$. miRNAs were added poly (A) tails by poly (A) polymerase (Ambion). The cDNAs were synthesized by a real-time primer, 5'-AACATGTACAGT CCATGGATGd(T)30N(A,G,C or T)-3'. miR-182 was then analyzed by qPCR and the primer used was: forward, 5'-GGC AATGGTAGAACTCACACT-3' and reverse, 5'-AACAT GTACAGTCCATGGATG-3'. qPCR analysis was performed using SuperTaq Polymerase (Takara Biotechnology Co., Ltd., Dalian, China). miR-182 expression was detected using the RG3000 system (Corbett Life Science, Mortlake, Australia) with the Quantitect SYBR-Green Kit (Qiagen, Hilden, Germany) as follows: initial denaturation at $95^{\circ} \mathrm{C}$ for $5 \mathrm{~min}$, followed by 40 cycles of $95^{\circ} \mathrm{C}$ denaturation for $20 \mathrm{sec}$, $52^{\circ} \mathrm{C}$ annealing for $20 \mathrm{sec}$ and extension at $72^{\circ} \mathrm{C}$ for $30 \mathrm{sec}$. Fluorescence was observed at $585 \mathrm{~nm}$ at each extension step of $72^{\circ} \mathrm{C}$. Human $5 \mathrm{~S}$ rRNA was added into each sample and served as a control. All experiments were repeated in triplicate.

Statistical analysis. Data were first tested for normal distribution and variance homogeneity using the Shapiro-Wilk test and F-test, respectively. Data are presented as mean \pm SD for normal distributions, otherwise, data are presented as median and quartiles. Since age, height and weight showed normal distributions, differences between these groups were analyzed using the Student's t-test. However, when the levels of miR-182 did not show a normal distribution, non-parametric tests were applied. miR-182 continuous variables between groups were analyzed by the Wilcoxon rank-sum test. Statistical analyses were performed using $\mathrm{R}$ version $2.15 .0^{\odot}$ (2012; ISBN 3-900051-07-0). $\mathrm{P}<0.05$ was considered to indicate a statistically significant difference.

\section{Results}

Clinical characteristics of patients. In total, 46 BC patients and 58 controls participated in the present study. The demographic and clinical characteristics of all the patients and controls are provided in Table I. No differences in age, height and weight were found between the BC patients and their controls. Of the 46 patients, 29 patients were ER-positive (63.0\%) and 28 PR-positive (60.9\%) in the entire tumor set (46 cases). Alcohol and passive smoking have been reported to increase BC risk (17-19), but no significant differences were identified between the BC patients and their controls in the present study (Table I).

Higher expression of miR-182 in BC tissues. miR-182, as an oncogene, is important for the development of BC $(20,21)$. To further demonstrate the role of miR-182 in BC, its expression was detected in the $\mathrm{BC}$ tissues. The results showed that miR-182 expression was markedly increased ( $>4$-fold higher) in $\mathrm{BC}$ tissues $(\mathrm{n}=3)$ compared with paracancerous tissues $(n=3)$ (Fig. 1), which is consistent with the oncogenic role of miR-182.

Higher levels of miR-182 in the serum of patients with BC. Furthermore, the serum levels of miR-182 were detected by $\mathrm{qPCR}$ to investigate the role of $\mathrm{miR}-182$ in the diagnosis of BC. It was found that the serum miR-182 levels in BC patients were $7.075 \times 10^{3}$ copies $/ \mathrm{ml}(\mathrm{n}=46)$, which were signifi- 
Table I. Demographic and clinical characteristics of the study samples.

\begin{tabular}{lccc}
\hline Characteristics & Healthy controls & Patients $^{\text {P-value }}$ \\
\hline $\mathrm{n}$ & 58 & 46 & 0.060 \\
Age (mean \pm SD), years & $52.00 \pm 9.81$ & $48.30 \pm 10.03$ & 0.400 \\
Weight (mean \pm SD), kg & $65.31 \pm 8.63$ & $66.00 \pm 8.65$ & 0.680 \\
Height (mean \pm SD), $\mathrm{cm}$ & $157.98 \pm 4.63$ & $158.76 \pm 4.64$ & - \\
ER-positive/negative, $\mathrm{n}$ & - & $29 / 17$ & - \\
PR-positive/negative, $\mathrm{n}$ & - & $28 / 18$ & 0.085 \\
Non-alcoholic/alcoholic drinks, $\mathrm{n}$ & $57 / 1$ & $41 / 5$ & 0.095 \\
Non-passive/passive smokers, $\mathrm{n}$ & $27 / 31$ & $14 / 32$ & $3.947 \mathrm{E}^{-08 \mathrm{~b}}$ \\
Median miR-182, $\mathrm{n}$ & $0.003^{\mathrm{b}}$ & $7.075^{\mathrm{b}}$ &
\end{tabular}

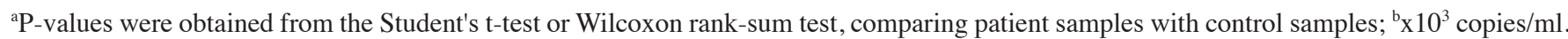
ER, estrogen receptor; PR, progesterone receptor.

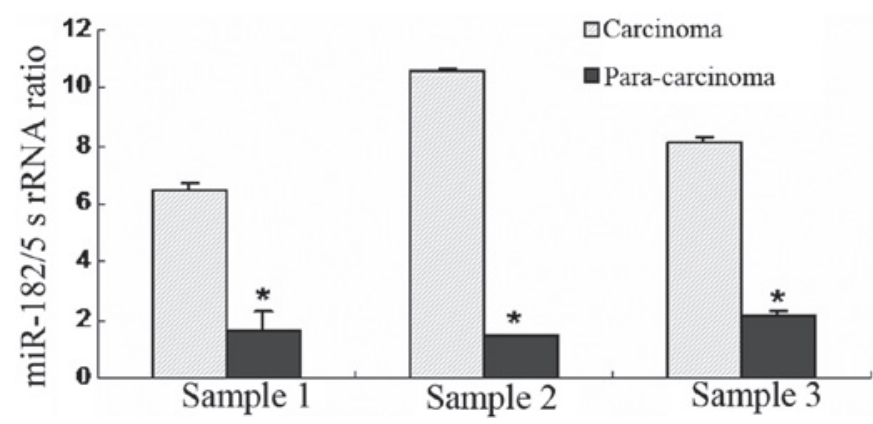

Figure 1. miR-182 expression in BC tissues. qPCR results showed that miR-182 expression was considerably higher in the $\mathrm{BC}$ tissues compared with control tissues $(\mathrm{P}<0.01)$. Samples $1-3$; three $\mathrm{BC}$ and para-carcinoma tissues. 5S rRNA was used as a control. BC, breast cancer; qPCR, quantitative PCR.

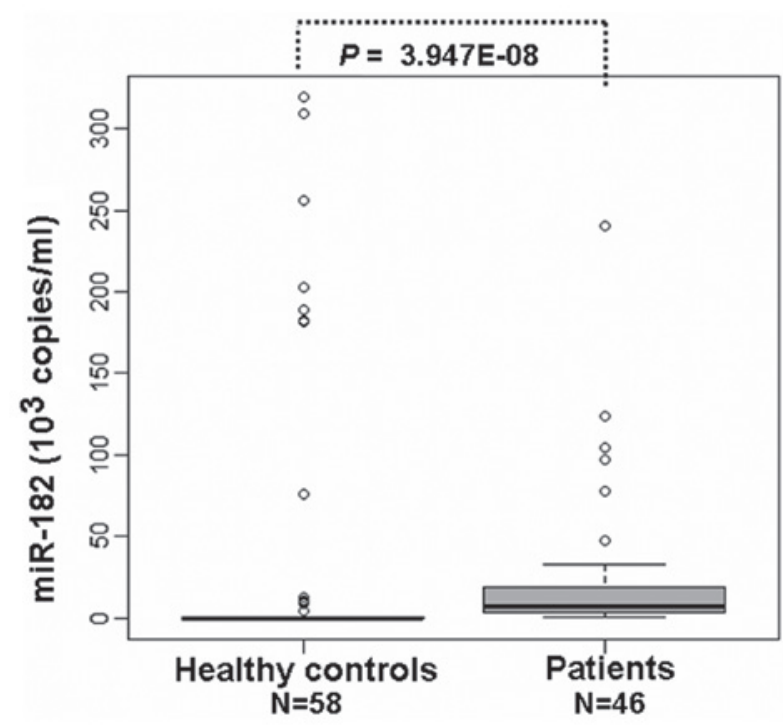

Figure 2. Serum miR-182 levels in BC patients and their controls. qPCR showed that serum miR-182 levels in BC patients $(n=46)$ were considerably higher compared with healthy controls $(\mathrm{n}=58)\left(\mathrm{P}=3.947 \mathrm{E}^{-08}\right)$. BC, breast cancer; qPCR, quantitative PCR.

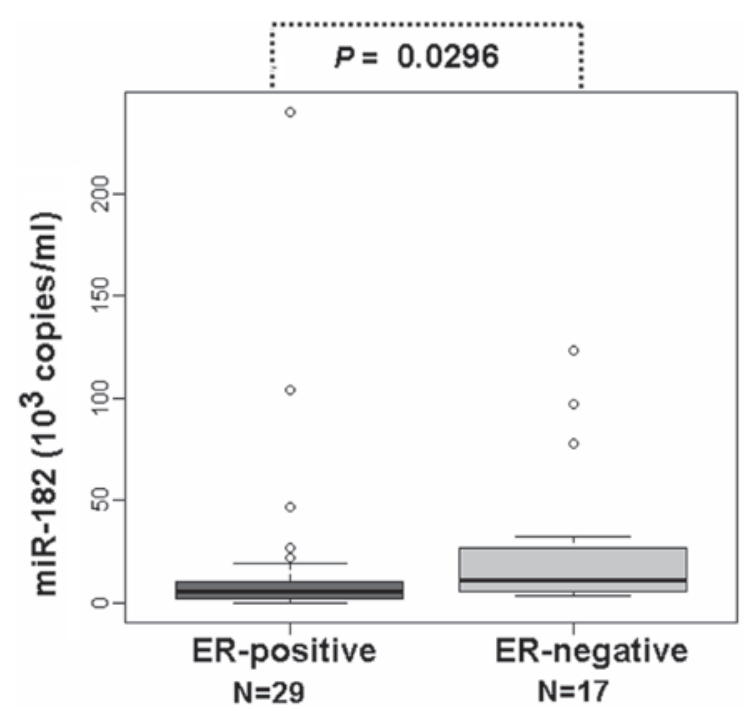

Figure 3. Correlation between serum miR-182 levels and ER-positive expression in BC patients. qPCR showed that serum miR-182 levels were considerably lower in ER-positive BC patients compared with ER-negative BC patients $(\mathrm{P}=0.0296)$. ER, estrogen receptor; $\mathrm{BC}$, breast cancer; $q \mathrm{PCR}$, quantitative PCR.

cantly higher compared with the serum of healthy controls $\left(0.003 \times 10^{3}\right.$ copies/ml $)(\mathrm{P}<0.01 ; \mathrm{n}=58$; Table I; Fig. 2$)$. The results demonstrated that the serum levels of miR-182 were higher in BC, indicating that miR-182 may also be an important factor for the pathogenesis of $\mathrm{BC}$.

Correlation of ER/PR with circulating miR-182 in the serum of $B C$ patients. ER and PR are important factors associated with the etiology and therapy of BC $(22,23)$. To study the correlation between ER and PR with the serum levels of miR-182, the serum levels of miR-182 were detected in ER- and PR-positive patients and compared with ER- and PR-negative patients. The results showed that the serum levels of miR-182 in the ER-positive patients $(n=29)$ were $5.41 \times 10^{3}$ copies $/ \mathrm{ml}$, considerably lower compared with the ER-negative patients $(n=17)(P<0.05$; Table II; Fig. 3$)$. The 
Table II. Correlation between ER- and PR-positive samples with miR-182.

\begin{tabular}{lccc}
\hline Receptor & $\mathrm{n}$ & Median miR-182 & P-value $^{\mathrm{a}}$ \\
\hline ER & & & \\
Positive & 29 & $10.409^{\mathrm{b}}$ & 0.0296 \\
Negative & 17 & & \\
PR & & $5.395^{\mathrm{b}}$ & 0.1130 \\
Positive & 28 & $10.643^{\mathrm{b}}$ & \\
Negative & 18 & & \\
\hline
\end{tabular}

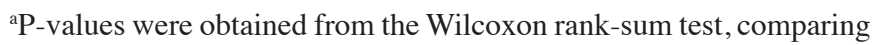
ER- and PR-positive samples with ER- and PR-negative samples; ${ }^{\mathrm{b}} \mathrm{X} 10^{3}$ copies $/ \mathrm{ml}$. ER, estrogen receptor; PR, progesterone receptor.

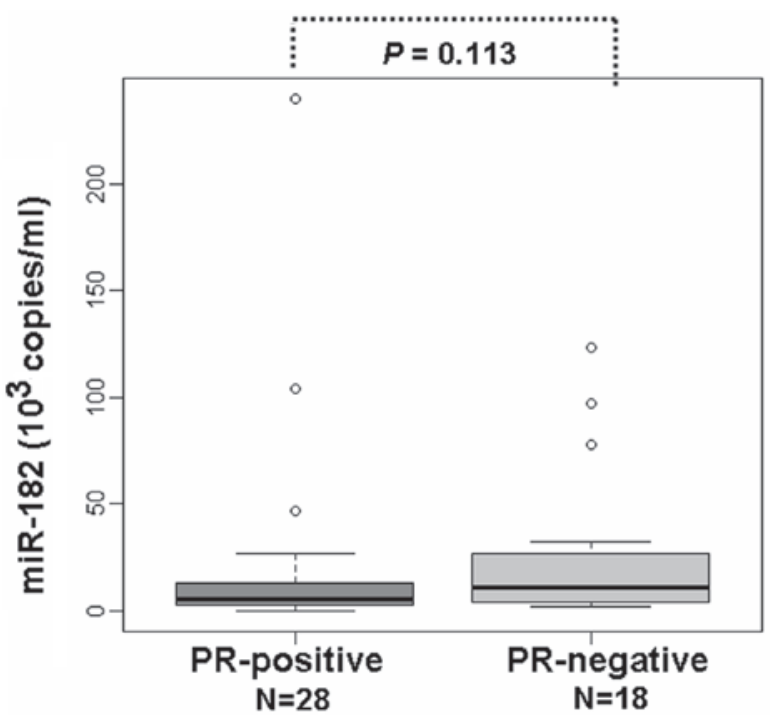

Figure 4. Correlation between serum miR-182 levels and PR-positive expression in BC patients. qPCR showed that serum miR-182 levels were lower in PR-positive BC patients compared with ER-negative BC patients, but there was no statistically significant difference. $\mathrm{PR}$, progesterone receptor; $\mathrm{BC}$, breast cancer; ER, estrogen receptor; qPCR, quantitative PCR.

serum levels of miR-182 in the PR-positive patients $(n=28)$ were also found to be lower compared with the PR-negative patients $(n=18)$ (Table II; Fig. 4).

\section{Discussion}

The ideal biomarkers for BC diagnosis should be easily accessible in order that they may be sampled relatively non-invasively. In addition, biomarkers must be sensitive enough to be detected in early stage tumors in almost all patients, while absent or minimal in healthy control individuals (24). miRNAs are markedly stable molecules, preserved well in formalin-fixed and fresh snap frozen specimens $(25,26)$. Their expression profiles are pathognomonic or tissue-specific in tumors (27), which establishes them as an ideal class of biomarker for BC diagnosis. In the current study, miR-182 was isolated from the tissues of BC patients and healthy controls and it was found that miR-182 expression was considerably higher in the $\mathrm{BC}$ tissues compared with the control tissues. This result is consistent with the oncogenic role of miR-182 in various types of cancer. Furthermore, miR-182 was isolated from the serum of BC patients and controls. The results demonstrated that miR-182 levels in the serum of BC patients were also markedly higher than those of the controls, indicating miR-182 is a useful biomarker for $\mathrm{BC}$ diagnosis.

Previous miRNA expression studies in BC have indicated the importance and potential roles of miRNA as disease classifiers and prognostic tools. Iorio et al (28) previously identified that 29 miRNAs were differentially expressed in BC tissues compared with control tissues. In addition, Mattie et al reported unique sets of miRNAs associated with BCs, which were defined by their roles of HER2/neu or ER/PR status (29). It has been previously reported that the pre-miR-27a rs895819 polymorphism may be associated with BC susceptibility or cancer development in Caucasians (30). The $\mathrm{C}$ allele of hsa-miR-146a (31) and hsa-miR-196a2 rs11614913 SNP (32), associated with $\mathrm{BC}$ risk, were also demonstrated to be important in familial breast/ovarian tumor development. These studies indicated that miRNAs are crucial in the development and diagnosis of BC.

Recently, several studies support that miR-182 acts as an oncogene in the development of BC $(20,21,33,34)$. miR-182 is overexpressed in human BC tissues and cell lines (MB-231 cells) and $\beta$-catenin binds to the promoter to increase the expression of miR-182 (20). Highly expressed miR-182 functions as a potential oncomir in BC (21), which disrupts the homologous recombination pathway in $\mathrm{BC}$ tissues. Mechanistically, the overexpression of miR-182 decreases BRCA1 protein levels and impedes DNA repair, while antagonizing miR-182 enhances BRCA1 levels and induces resistance to the poly (ADP-ribose) polymerase 1 inhibitor (33). FOXO1, a putative tumor suppressor, is also a target of miR-182 and an antisense inhibitor specific to miR-182 which leads to a significant increase in endogenous FOXO1 expression (34). Similarly, the current study demonstrated that miR-182 was upregulated in BC tissues compared with control tissues, consistent with the important role of miR-182 in the tumorigenesis of BC.

miRNAs have been previously demonstrated to be present in the serum in a stable and reproducible manner. In addition, the unique expression patterns of serum miRNAs may be used as biomarkers for various diseases $(13,35)$. Using qPCR, miR-205 was demonstrated to be downregulated, while miR-155 was upregulated in BC patient serum (36). The plasma levels of circulating miR-10b and miR-373 were found to be significantly higher in $\mathrm{BC}$ patients with lymph node metastasis compared with normal controls (37). In contrast to increased miR-21 levels, circulating miR-92a levels were decreased in the BC patients compared with the controls (38). Although miR-182 has been reported to be important for BC tumorigenesis, no previous studies have analyzed the role of circulating miR-182 in the diagnosis of $\mathrm{BC}$. To explore the diagnostic role of circulating miR-182 in $\mathrm{BC}$, the present study isolated miRNAs from the serum of $\mathrm{BC}$ patients and healthy control individuals. The results revealed that the miR-182 levels in $\mathrm{BC}$ were higher 
compared with the healthy controls, which confirmed the diagnostic role of miR-182 in BC.

The prognostic and therapeutic roles of ER or PR in BC have been studied extensively and are well established $(22,23,39)$. Significant associations have been found between ER- and PR-positive rates with menopausal status, tumor size or the presence of distant metastases in BCs (40). In total, $>75 \%$ of primary BC patients express ER and $\sim 50 \%$ of these tumors are stained positively with PR4. The results of the present study also showed that $64.4 \%$ of primary BC patients express ER and $62.2 \%$ of these patients PR. The effects of ER- and PR-positive expression on the serum levels of miR-182 were further investigated and it was found that serum levels of miR-182 were lower in the ER- and PR-positive patients compared with the ER- and PR-negative subjects. The results indicated that there is a close correlation between serum levels of miR-182 and ERand PR-positive expression in BC patients. Although alcohol and passive smoking increases BC risk (17-19), in the present study, no significant differences were identified between the $\mathrm{BC}$ patients and their controls. The relatively small number of available previous studies may lead to this limitation.

In summary, the results of the present study showed that the levels of miR-182 in the serum of BC patients were upregulated compared with healthy controls. Notably, the levels of miR-182 in the serum of ER-positive patients was considerably lower compared with the ER-negative patients. Overall, the present study highlights miR-182 as a novel diagnostic marker for BC.

\section{Acknowledgements}

The present study was supported by the NCET-10-0919, the National Natural Science Foundation (no. 31371321, 81141114 and 81200601) and the Foundation of Shandong Educational Committee of China (no. J10LC60 and J11LC01).

\section{References}

1. Sariego J: Breast cancer in the young patient. Am Surg 76 1397-1400, 2010.

2. Anders CK and Carey LA: Biology, metastatic patterns, and treatment of patients with triple-negative breast cancer. Clin Breast Cancer 9 (Suppl 2): S73-S81, 2009.

3. Kloosterman WP and Plasterk RH: The diverse functions of microRNAs in animal development and disease. Dev Cell 11: 441-450, 2006.

4. Stefani G and Slack FJ: Small non-coding RNAs in animal development. Nat Rev Mol Cell Biol 9: 219-230, 2008.

5. Di Leva G, Piovan C, Gasparini P, et al: Estrogen mediated-activation of miR-191/425 cluster modulates tumorigenicity of breast cancer cells depending on estrogen receptor status. PLoS Genet 9: e1003311, 2013

6. Ozgün A, Karagoz B, Bilgi O, Tuncel T, Baloglu H and Kandemir EG: MicroRNA-21 as an indicator of aggressive phenotype in breast cancer. Onkologie 36: 115-118, 2013.

7. Han M, Liu M, Wang Y, et al: Re-expression of miR-21 contributes to migration and invasion by inducing epithelial-mesenchymal transition consistent with cancer stem cell characteristics in MCF-7 cells. Mol Cell Biochem 363: 427-436, 2012.

8. Yu SJ, Hu JY, Kuang XY, et al: MicroRNA-200a promotes anoikis resistance and metastasis by targeting YAP1 in human breast cancer. Clin Cancer Res 19: 1389-1399, 2013.

9. Castilla MÁ, Díaz-Martín J, Sarrió D, et al: MicroRNA-200 family modulation in distinct breast cancer phenotypes. PLoS One 7: e47709, 2012.

10. Sun X, Qin S, Fan C, Xu C, Du N and Ren H: Let-7: a regulator of the ER $\alpha$ signaling pathway in human breast tumors and breast cancer stem cells. Oncol Rep 29: 2079-2087, 2013.
11. Taylor DD and Gercel-Taylor C: MicroRNA signatures of tumor-derived exosomes as diagnostic biomarkers of ovarian cancer. Gynecol Oncol 110: 13-21, 2008.

12. Hunter MP, Ismail N, Zhang X, et al: Detection of microRNA expression in human peripheral blood microvesicles. PLoS One 3: e3694, 2008.

13. Mitchell PS, Parkin RK, Kroh EM, et al: Circulating microRNAs as stable blood-based markers for cancer detection. Proc Natl Acad Sci USA 105: 10513-10518, 2008.

14. Zhu W, Qin W, Atasoy U and Sauter ER: Circulating microRNAs in breast cancer and healthy subjects. BMC Res Notes 2: 89, 2009.

15. Heneghan HM, Miller N, Lowery AJ, Sweeney KJ, Newell J and Kerin MJ: Circulating microRNAs as novel minimally invasive biomarkers for breast cancer. Ann Surg 251: 499-505, 2010.

16. Schrauder MG, Strick R, Schulz-Wendtland R, et al: Circulating micro-RNAs as potential blood-based markers for early stage breast cancer detection. PLoS One 7: e29770, 2012.

17. Wu AH, Vigen C, Razavi P, Tseng CC and Stancyzk FZ: Alcohol and breast cancer risk among Asian-American women in Los Angeles County. Breast Cancer Res 14: R151, 2012.

18. Shrubsole MJ, Gao YT, Dai Q, et al: Passive smoking and breast cancer risk among non-smoking Chinese women. Int J Cancer 110: 605-609, 2004.

19. Gammon MD, Eng SM, Teitelbaum SL, et al: Environmental tobacco smoke and breast cancer incidence. Environ Res 96: 176-185, 2004.

20. Chiang $\mathrm{CH}$, Hou MF and Hung WC: Up-regulation of miR-182 by $\beta$-catenin in breast cancer increases tumorigenicity and invasiveness by targeting the matrix metalloproteinase inhibitor RECK. Biochim Biophys Acta 1830: 3067-3076, 2013.

21. Krishnan K, Steptoe AL, Martin HC, et al: MicroRNA-182-5p targets a network of genes involved in DNA repair. RNA 19: 230-242, 2013

22. Cui X, Schiff R, Arpino G, Osborne CK and Lee AV: Biology of progesterone receptor loss in breast cancer and its implications for endocrine therapy. J Clin Oncol 23: 7721-7735, 2005.

23. Diaz NM: Laboratory testing for HER $2 / n$ eu in breast carcinoma: an evolving strategy to predict response to targeted therapy. Cancer Control 8: 415-418, 2001.

24. Heneghan HM, Miller N, Lowery AJ, Sweeney KJ and Kerin MJ: MicroRNAs as Novel Biomarkers for Breast Cancer. J Oncol 2009: 950201, 2009.

25. Xi Y, Nakajima G, Gavin E, et al: Systematic analysis of microRNA expression of RNA extracted from fresh frozen and formalin-fixed paraffin-embedded samples. RNA 13: 1668-1674, 2007.

26. Li J, Smyth P, Flavin R, et al: Comparison of miRNA expression patterns using total RNA extracted from matched samples of formalin-fixed paraffin-embedded (FFPE) cells and snap frozen cells. BMC Biotechnol 7: 36, 2007.

27. Lu J, Getz G, Miska EA, et al: MicroRNA expression profiles classify human cancers. Nature 435: 834-838, 2005.

28. Iorio MV, Ferracin M, Liu CG, et al: MicroRNA gene expression deregulation in human breast cancer. Cancer Res 65 : 7065-7070, 2005.

29. Mattie MD, BenzCC, Bowers J, et al: Optimized high-throughput microRNA expression profiling provides novel biomarker assessment of clinical prostate and breast cancer biopsies. Mol Cancer 5: 24, 2006.

30. Zhong S, Chen Z, Xu J, Li W and Zhao J: Pre-mir-27a rs895819 polymorphism and cancer risk: a meta-analysis. Mol Biol Rep 40: 3181-3186, 2013

31. Pastrello C, Polesel J, Della Puppa L, Viel A and Maestro R: Association between hsa-mir-146a genotype and tumor age-of-onset in BRCA1/BRCA2-negative familial breast and ovarian cancer patients. Carcinogenesis 31: 2124-2126, 2010.

32. Linhares JJ, Azevedo M Jr, Siufi AA, et al: Evaluation of single nucleotide polymorphisms in microRNAs (hsa-miR-196a2 rs11614913 $\mathrm{C} / \mathrm{T}$ ) from Brazilian women with breast cancer. BMC Med Genet 13: 119, 2012.

33. Moskwa P, Buffa FM, Pan Y, et al: miR-182-mediated downregulation of BRCA1 impacts DNA repair and sensitivity to PARP inhibitors. Mol Cell 41: 210-220, 2011.

34. Guttilla IK and White BA: Coordinate regulation of FOXO1 by miR-27a, miR-96, and miR-182 in breast cancer cells. J Biol Chem 284: 23204-23216, 2009. 
35. Chen X, Ba Y, Ma L, et al: Characterization of microRNAs in serum: a novel class of biomarkers for diagnosis of cancer and other diseases. Cell Res 18: 997-1006, 2008.

36. Liu J, Mao Q, Liu Y, Hao X, Zhang S and Zhang J: Analysis of miR-205 and miR-155 expression in the blood of breast cancer patients. Chin J Cancer Res 25: 46-54, 2013.

37. Chen W, Cai F, Zhang B, Barekati Z and Zhong XY: The level of circulating miRNA-10b and miRNA-373 in detecting lymph node metastasis of breast cancer: potential biomarkers. Tumour Biol 34: 455-462, 2013.

38. Si H, Sun X, Chen Y, et al: Circulating microRNA-92a and microRNA-21 as novel minimally invasive biomarkers for primary breast cancer. J Cancer Res Clin Oncol 139: 223-229, 2013.
39. Osborne CK, Schiff R, Arpino G, Lee AS and Hilsenbeck VG: Endocrine responsiveness: understanding how progesterone receptor can be used to select endocrine therapy. Breast 14: 458-465, 2005

40. Faheem M, Mahmood H, Khurram M, Qasim U and Irfan J: Estrogen receptor, progesterone receptor, and Her 2 Neu positivity and its association with tumour characteristics and menopausal status in a breast cancer cohort from northern Pakistan. Ecancermedicalscience 6: 283, 2012. 